\title{
COVID-19 pandemic impacts physical activity levels and sedentary time but not sleep quality in young badminton athletes
}

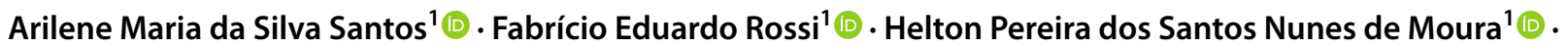

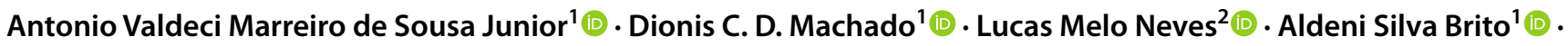

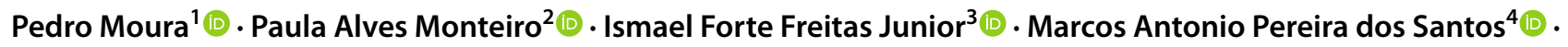 \\ Sérgio Luiz Galan Ribeiro' ${ }^{10}$
}

Received: 2 February 2021 / Accepted: 8 April 2021 / Published online: 21 April 2021

(c) The Author(s), under exclusive licence to Springer-Verlag Italia S.r.l., part of Springer Nature 2021

\begin{abstract}
Purpose Regular physical activity is a good strategy to maintain the health of athletes, and prevent pain and decreased joint flexibility during the pandemic. On the other hand, higher sedentary time during the pandemic period can have deleterious effects. The objective of this study was to compare physical activity levels, sedentary time, and sleep parameters during the pre-COVID period and the COVID-19 pandemic period in young badminton athletes.

Methods Fifteen young badminton athletes were evaluated during a pre-COVID period (July 2019) and during the COVID19 period (July 2020). Sleep parameters, physical activity level, and sedentary time were measured using a tri-axial accelerometer. Participants wore the accelerometer on their dominant wrist for 7 days consecutively. In addition, the average of each sleep parameter [time in bed and total sleep time in hours per day, sleep efficiency (\%), wake after sleep onset (WASO, total per day), and sleep latency (minutes per day)] was reported over the 7-day period.

Results Athletes presented increased sedentary time (pre-COVID $=7.0 \pm 1.1 \mathrm{vs}$.COVID-19=8.9 $\pm 1.9 \mathrm{~h} / \mathrm{day}, p=0.004$, $d=1.30$ ) and significant decreases in the total PA observed in counts per day (pre-COVID $=2,967,064.4 \pm 671,544.1 \mathrm{vs}$. COVID-19 $=1,868,210.2 \pm 449,768.4$ counts/day, $p=0.001, d=1.99$ ), time in vigorous PA (pre-COVID $=7.7 \pm 0.9$ vs. COVID-19 $=6.1 \pm 1.2 \mathrm{~h} /$ day, $p=0.001, d=1.56$ ), and time in moderate-to-vigorous PA (pre-COVID $=8.1 \pm 0.9$ vs. COVID$19=6.5 \pm 1.3 \mathrm{~h} /$ day, $p=0.001, d=1.48$ ). There were no significant differences for time in light and moderate PA or in sleep parameters $(p>0.05)$.

Conclusion Young badminton athletes presented increased sedentary time, and decreased total physical activity, time in MVPA, and time in vigorous activities during the COVID-19 pandemic compared to the pre-COVID period, however, there were no significant differences in sleep parameters.
\end{abstract}

Keywords COVID-19 pandemic $\cdot$ Athletes $\cdot$ Physical activity $\cdot$ Sedentary behavior $\cdot$ Sleep

Fabrício Eduardo Rossi

fabriciorossi@ufpi.edu.br

1 Immunometabolism of Skeletal Muscle and Exercise Research Group, Department of Physical Education, Federal University of Piauí (UFPI), Ministro Petrônio Portella" Campus, Teresina, PI 64049-550, Brazil

2 Department of Psychiatry, Post-Graduation Program in Health Sciences, Santo Amaro University, Sao Paulo, Brazil and Bipolar Disorder Program (PROMAN), University of Sao Paulo Medical School, São Paulo, Brazil

3 Department of Physical Education, São Paulo State University (UNESP), Presidente Prudente, São Paulo, Brazil

4 Department of Biophysics and Physiology, Federal University of Piauí (UFPI), Campus Minister Petrônio Portela, Teresina, PI, Brazil

\section{Introduction}

Although athletes comply with the World Health Organization (WHO) [1] recommendations of physical activity (PA) for health (150 min a week of moderate-vigorous PA or 75 min a week of vigorous-very vigorous PA), since they often perform high-intensity daily training routines, this routine represents only part of the athlete's day, with twothirds of the day being composed of low-intensity activities or sedentary time (ST) [2]. Sedentary behaviors (SB) are conceptualized as activities with energy expenditure $\leq 1.5$ metabolic equivalents (MET), such as watching television, using a computer or smartphone, and playing video games 
[3], SB are associated with impaired glucose metabolism [4], cardiovascular diseases and mortality [5], and low physical fitness [6]. Weiler et al.[7] verified that $79 \%$ of the daily routine of English first division professional football players was characterized by SB. Sperlich et al. [5] demonstrated that rowers of the German elite, despite complying with the time recommendations for moderate-vigorous PA, remained in SB for more than $11.5 \mathrm{~h}$ a day. Thus, although highly trained athletes accomplish the moderate-to-vigorous PA recommendations, they present an amount of SB similar to individuals who do not meet the recommendations.

In addition, the COVID-19 pandemic, described as an acute respiratory syndrome caused by coronavirus 2 , has restricted PA and increased SB in people of all ages [8], especially due to the necessity of social isolation and, in some cases, lockdown, the principal strategy adopted to decrease contamination $[9,10]$. In fact, 2 meta-analysis with more than 90,000 subjects, showed a decrease in PA and an increase in SB [11, 12] during social isolation and lockdown periods around the world. Highly trained athletes need to adapt, or in some cases stop, their regular training sessions and competitive routine for several weeks - which is likely to decrease their strength and endurance performance after detraining [13], inducing possible degeneration of all the structures of a joint [14], during social isolation for COVID19, which can change their habitual PA and SB, and lead to lower sleep quality [15]. In non-athlete populations, the association of a reduction in PA [16-18] and increased SB [19] with lower sleep quality is well accepted.

Sleep quality has huge importance, since it enables maintained alertness, contributes to a shorter reaction time, and improves mood and physical fitness [20]. Lastella et al. [21] demonstrated that, in professional soccer players, sleep quality after a match is worse than after a training day, and that a trip to compete in another country can impair the quality and quantity of sleep; therefore, sleep quality and quantity seem to be influenced by environmental changes. Differences between sports disciplines such as triathlon, volleyball, and soccer [22] can affect the sleep quality of athletes. In addition, the form of stimulus (which has been impacted during the pandemic), such as high-intensity interval training or small-sided game training [23], can also affect the sleep quality of athletes. Therefore, the training and sleep quality of athletes are known to be complex issues [23, 24], and comparing the pandemic period with the period prior to the pandemic is relevant in badminton athletes.

There are no studies in the literature that investigate the impact of the COVID-19 pandemic (no pandemic period vs pandemic period) using an objective measure of PA, ST, and sleep parameters in young trained athletes. In this way, the current study aimed to compare the levels of habitual PA, ST, and sleep quantity and quality of badminton athletes during the COVID-19 pandemic period and no pandemic period ( 1 year before the pandemic) when they were training regularly for the main competition of that year. We hypothesized that athletes during the COVID-19 pandemic period would show worse sleep parameters and PA levels, as well as higher ST, compared to the no pandemic period.

\section{Methods}

\section{Subjects}

The sample consisted of 15 young badminton athletes recruited for convenience through contact with coaches of the Brazilian Badminton Confederation, nine women $($ age $=18.5 \pm 2.6$ years $)$, and 6 men $($ age $=18.7 \pm 1.8$ years $)$, according to the characteristics shown in Table 1. The inclusion criteria in the study were: being over 15 years old at the date of collection; not presenting injuries during the evaluation period; performing the daily training routine during the evaluation week; and signing the informed consent form. Athletes were excluded if they did not wear the accelerometer appropriately.

All athletes had at least 2 years of experience in badminton and participated in national and international competitions. The assessments were performed in 16 athletes during a pre-COVID period (June 2019) when they were training consistently (5-6 times a week, about $3 \mathrm{~h}$ a day), 2 weeks before the Brazilian Badminton championship, which was the main competition for these athletes in that year. Twelve months later, during the COVID-19 pandemic (June 2020), 15 athletes were re-evaluated. The one dropout was due to an athlete who missed the assessment during the pandemic.

According to the state laws during the pandemic, Brazilian citizens and athletes are required to adhere to local laws and social isolation. Athletes needed to stop their regular training routine in March 2020, thus, at the time of this study, they had not participated in their regular routine for three months. During the pandemic, athletes were encouraged by coaches to train individually at home to keep themselves fit and healthy, focusing only on physical capabilities, such as flexibility, aerobic capacity, and agility, and not on

Table 1 Characteristics of the athletes

\begin{tabular}{lrrc}
\hline Variables & Total $(n=15)$ & Male $(n=6)$ & Female $(n=9)$ \\
\hline Height $(\mathrm{cm})$ & $167.5 \pm 8.4$ & $175.5 \pm 4.6$ & $163.1 \pm 6.5^{*}$ \\
Body weight $(\mathrm{kg})$ & $63.5 \pm 8.0$ & $69.5 \pm 5.9$ & $60.1 \pm 7.2^{*}$ \\
BMI $\left(\mathrm{kg} / \mathrm{m}^{2}\right)$ & $22.6 \pm 1.7$ & $22.6 \pm 2.3$ & $22.5 \pm 1.4$ \\
Badminton experience & $8.3 \pm 2.7$ & $9.0 \pm 1.8$ & $7.9 \pm 3.1$ \\
$\quad(\mathrm{y})$ & & & \\
\hline
\end{tabular}

$B M I$ body mass index. Values are mean \pm standard deviation. $*=$ significant difference between sexes 
technical or tactical training. The couches used video classes three times a week for about $60 \mathrm{~min}$ per day to guide their athletes and motivate them.

All participants or parents (when age $<18$ years) signed a consent form and were informed about the purpose of the study. Protocols were developed according to the Declaration of Helsinki and approved by the Research Ethics Committee at the Federal University of Piauí (protocol: 2.552.506).

\section{Procedures}

\section{Accelerometer}

\section{Habitual physical activity levels and sedentary time assessment}

Actigraph GT3X + accelerometers (ActiGraph, Pensacola FL, United States) were used to monitor physical activity and sleep [25]. Participants were encouraged to wear the accelerometer on their dominant wrist for 7 consecutive days, removing it only during showering or swimming. The minimum accelerometer data considered acceptable for analytical purposes were at least 4 days ( 3 weekdays and 1 weekend day), with a minimum of $10 \mathrm{~h}$ per day. Wear time for PA and ST excluded sleep time, as well as periods for which the activity monitor was removed (non-wear time). Data were collected at a sampling rate of $100 \mathrm{~Hz}$, downloaded as raw data and processed as 10 -s epochs. The total counts were recorded and the following cut-points were established: between 0 and 275 counts per $5 \mathrm{~s}$, as sedentary time, from 276 to 415 counts per $5 \mathrm{~s}$ as light PA, from 416 to 777 counts per $5 \mathrm{~s}$ as moderate PA, $\geq 778$ counts per $5 \mathrm{~s}$ as vigorous $\mathrm{PA}$, and $\geq 416$ counts per $5 \mathrm{~s}$ as moderate-tovigorous PA (MVPA), according to Crouter et al. [26] for analyzing dominant wrist accelerometer data in young people, since to our knowledge, there are no specific cut-points for badminton players.

\section{Sleep parameters}

Participants were encouraged to wear the accelerometer on their dominant wrist for 7 consecutive days and free-living sleep was analyzed by 1-min counts. The devices contain motion-sensitive accelerometers which have been previously validated for objective sleep measurement [27] and have been used consistently to monitor sleep in athletes [21, 28, 29]. Rest and sleep periods were automatically identified using the Actilife software from Actigraph (Pensacola, FL, USA) (version 6.13.0.), as well as through a hand-written sleep diary that each subject kept to record the time in bed and out of bed. The following parameters were reported for each sleep event over the 7-day period using the Actilife software: sleep efficiency (\%), time in bed and sleep time in hours per day, wake after sleep onset (total per day), and sleep latency (minutes per day). Total sleep time (hours) and sleep efficiency (ratio of sleep time to total time in bed $\times 100 ; \%$ ) were determined for every night using the Cole-Kripke automatic sleep/wake identification algorithm, where sleep is classified based on activity levels and a sleep/ wake calculation.

\section{Anthropometric assessment}

Body mass was measured using an electronic scale (Filizola PL 50, Filizola Ltda, Brazil), with an accuracy of $0.1 \mathrm{~kg}$ and a maximum capacity of $150 \mathrm{~kg}$, and stature was measured using a fixed stadiometer of the Sanny brand (Sanny, São Paulo, Brazil), with an accuracy of $0.1 \mathrm{~cm}$ and a maximum extension of two meters. The athletes remained barefoot, wearing light clothing, and standing at the base of the stadiometer, with their backs to the machine, touching their shoulder blades, buttocks, and heels to the equipment's vertical support. The body mass index (BMI) was calculated using $\left[\mathrm{BMI}=\right.$ body mass $(\mathrm{kg}) /$ stature $\left.\left(\mathrm{m}^{2}\right)\right]$.

\section{Statistical analysis}

The Shapiro-Wilk test was used to verify the normality of the data set and the data are shown as mean and standard deviation or median and interquartile range. The paired $t$ test was conducted to compare parametric variables between the pre-COVID and COVID-19 periods and the Wilcoxon test was applied when data presented non-parametric distribution. The effect size was calculated via Cohen's $d$, whereby a value of $>0.20$ was considered small, $>0.50$ moderate, and $>0.80$ large, and the $95 \%$ Confidence Intervals are reported $(95 \% \mathrm{CI})$. Statistical significance was set at $p<0.05$. The data were analyzed using the Statistical Package for Social Sciences 17.0 (SPSS Inc. Chicago. IL. USA).

\section{Results}

Table 1 shows the characteristics of the athletes, according to sex. Males presented greater height $(p=0.003)$ and body weight $(p=0.028)$ than females, however, there was no significant difference for BMI $(p=0.947)$. The athletes had mean badminton experience of 8.3 years and were in the top ten in their main category, according to the Brazilian badminton ranking published in December 2019.

Table 2 and Fig. 1 show the comparisons between sedentary time, total physical activity in counts per day, and levels of physical activity during the pre-COVID and COVID-19 periods in young trained athletes. 
Table 2 Comparison between sedentary time, total physical activity in counts per day, and levels of physical activity during the pre-COVID and COVID-19 periods in young trained athletes

\begin{tabular}{lllll}
\hline Variables & pre-COVID & COVID & $p$ & $E S$ \\
\hline Sedentary time (hour/day) & $6.7(0.9)$ & $8.8(1.9)$ & 0.004 & 1.30 \\
Time in light PA (hour/day) & $0.43 \pm 0.07$ & $0.46 \pm 0.10$ & 0.213 & 0.36 \\
Time in moderate PA (hour/day) & $0.43 \pm 0.05$ & $0.46 \pm 0.09$ & 0.271 & 0.43 \\
Time in vigorous PA (hour/day) & $7.7 \pm 0.9$ & $6.1 \pm 1.2$ & 0.001 & 1.56 \\
Time in MVPA (hour/day) & $8.1 \pm 0.9$ & $6.5 \pm 1.3$ & 0.001 & 1.48 \\
Total counts (counts/day) & $2,967,064.4 \pm 671,544.1$ & $1,868,210.2 \pm 449,768.4$ & 0.001 & 1.99 \\
\hline
\end{tabular}

MVPA moderate-to-vigorous physical activity, ES effect size. Values are mean \pm standard deviation or median (interquartile range)
The mean days analyzed for habitual physical activity levels and ST were $6.6 \pm 1.7$ days during the pre-COVID period and $6.8 \pm 0.4$ days in the COVID-19 period.

Athletes presented increases in ST of approximately $2 \mathrm{~h}$ during the pandemic ( $p=0.004, d=01.30,95 \%$ $\mathrm{CI}=-1.804$ to -0.730 ) and there was a significant decrease in the total physical activity observed in counts per day $(p=0.001, d=1.99,95 \% \mathrm{CI}=-197,574.3$ to $197,578.3)$.

Regarding the levels of physical activity, there were significant decreases for time in vigorous activities $(p=0.001$, $d=1.56,95 \% \mathrm{CI}=1.195-1.928)$ and moderate-to-vigorous physical activity ( $p=0.001, d=1.48,95 \% \mathrm{CI}=1.095-1.868)$ in relation to the pre-COVID period, with large effect sizes.

There were no significant differences in time spent in light PA ( $p=0.213, d=0.36,95 \% \mathrm{CI}=-0.390$ to -0.330$)$ and in moderate PA $(p=0.271, d=0.43,95 \% \mathrm{CI}=-0.452$ to -0.401 ). The effect sizes were small for both variables. In addition, there were no significant differences between sexes in ST and physical activity levels.

Table 3 and Fig. 2 present the comparisons between quantity and quality of sleep during the pre-COVID and COVID19 periods in young trained athletes. The average sleep event over the 7 days (Sunday to Saturday) was used to analyze sleep quantity and quality.

Athletes presented increased time in bed during the pandemic $(p=0.103)$ and decreased sleep efficiency $(p=0.233)$, but without significant differences between the periods analyzed. However, the effect sizes were moderate $(d=0.54$, $95 \% \mathrm{CI}=-0.937$ to -0.142$)$ and small $(d=0.27,95 \%$ $\mathrm{CI}=-2.640$ to 3.181 ), respectively.

There were no significant differences between periods for WASO $(p=0.078)$, sleep latency $(p=0.859)$, and sleep time $(p=0.454)$, although the effect size was moderate for WASO $(d=0.61,95 \% \mathrm{CI}=-14.921$ to 13.706$)$ and small for sleep latency $(d=0.17,95 \% \mathrm{CI}=-1.840$ to 1.497$)$ and sleep time $(d=0.17,95 \% \mathrm{CI}=-0.587$ to 0.242$)$. Furthermore, there were no significant differences between sexes in the sleep quantity and quality.

When analyzing the hand-written sleep diary that each athlete recorded, the athletes usually went to bed $\sim 2 \mathrm{~h}$ later and woke up $3 \mathrm{~h}$ later during the COVID period compared to the pre-COVID period.

\section{Discussion}

To our knowledge, this is the first study to compare total physical activity, sedentary time, and sleep parameters using an objective measurement during the pre-COVID period and the COVID-19 pandemic in young badminton athletes. The main findings of this study were that young badminton athletes presented increased ST, and decreased total physical activity observed in counts per day, and time in MVPA and vigorous activities during the COVID-19 pandemic compared to the pre-COVID period. However, although the athletes increased their time in bed by approximately $1 \mathrm{~h}$ during the quarantine, there were no significant differences in quantity and quality of sleep.

Badminton is the fastest racket sport, with the aerobic system being responsible for approximately $60-70 \%$ of the actions and the anaerobic system for approximately $30 \%$ [30]. The sport requires speed, agility, and effective decision-making, as well as anticipating opponents' movements, quick changes in direction, jumps, and frequent stop-and-go actions that demand power and muscular endurance [30, 31]. During the pandemic, highly trained athletes stopped their regular training sessions and competitive routine for several weeks, which may decrease their strength and endurance performance after detraining [13], added to which, COVID19 has physical, nutritional, and psychological consequences that may impact the safe return to sport and general health of athletes [32]. Thus, it is extremely important to analyze daily life activities in this population to prevent their performance from being impaired in the future.

In the current study, athletes showed higher ST during social isolation in relation to the pre-COVID period ( 27\%). Paoli and Musumeci [14] showed that higher ST could decrease performance in athletes, reducing maximum oxygen consumption (VO2max), strength, and endurance capacity, regardless of their previous physical fitness condition [13]. Furthermore, lower time in sedentary breaks and 


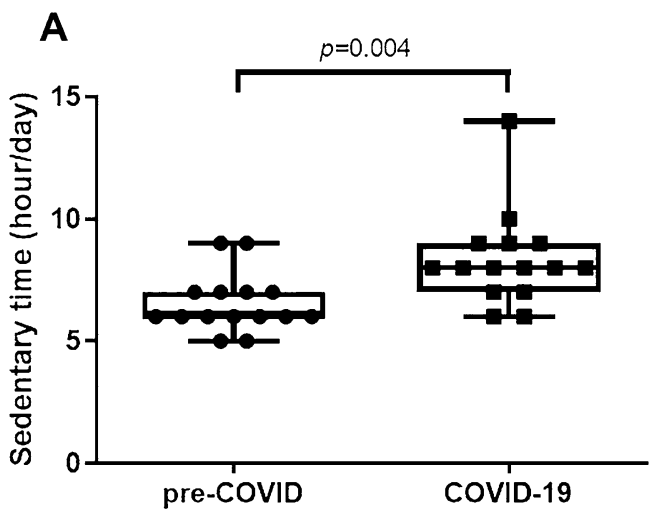

B
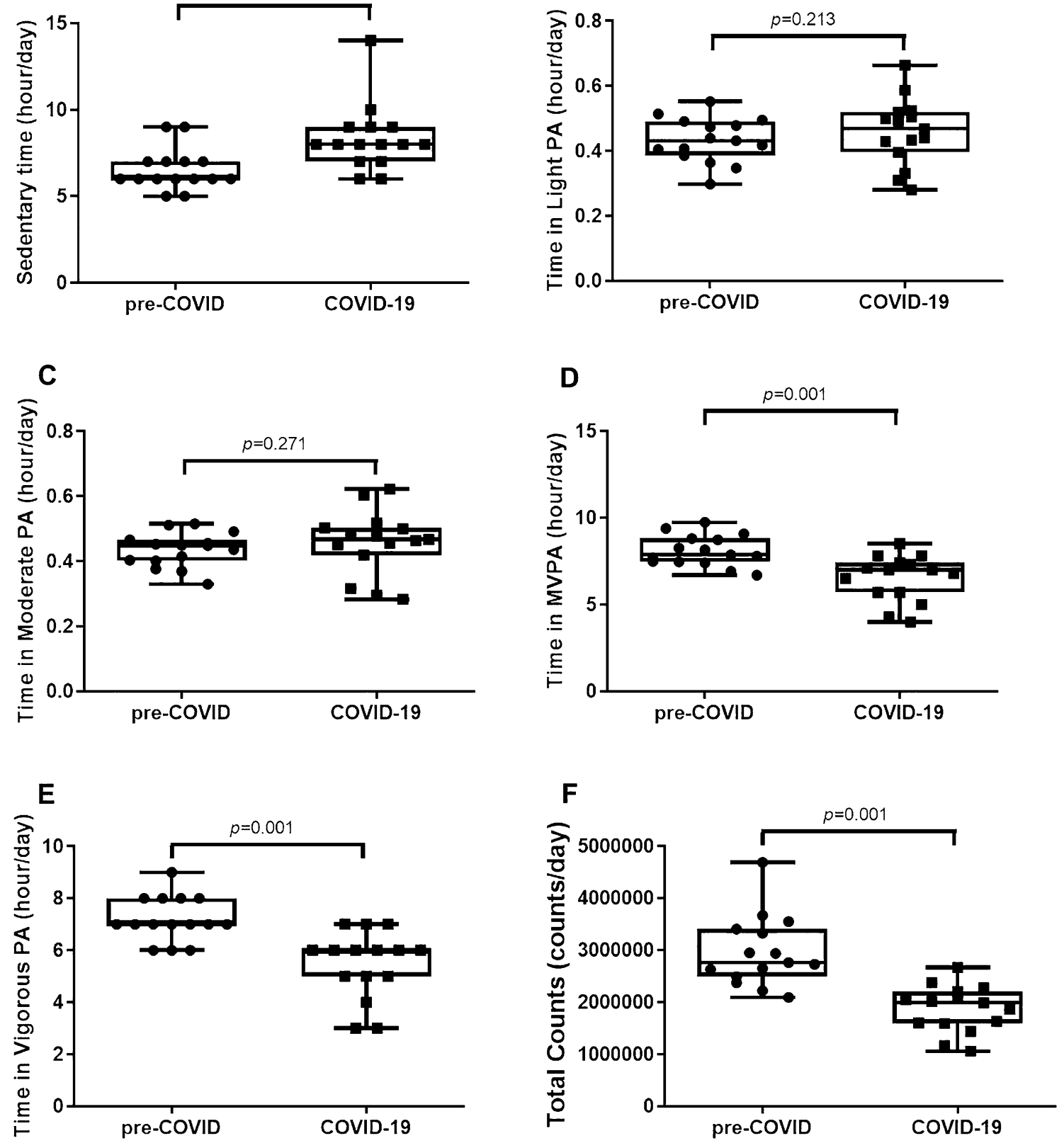

Fig. 1 Comparison between sedentary time, levels of physical activity, and total physical activity in counts per day during the preCOVID and COVID-19 periods in young trained athletes. a Sedentary time (hours per day) in median and interquartile range; $\mathbf{b}$ Time in light physical activity (hours per day) in mean and standard deviation;

c Time in moderate physical activity (hours per day) in mean and standard deviation; $\mathbf{d}$ Time in moderate-to-vigorous physical activity in mean and standard deviation; e Time in vigorous physical activity (hours per day) in mean and standard deviation; f Total counts (counts per day) in mean and standard deviation

less skeletal muscle activity can increase the risk of muscle-tendon injuries and also impair metabolic mechanisms associated with glucose and triglyceride absorption [33]. In this sense, decreased ST and increased sedentary breaks are extremely important for people of all ages and populations.

Although the athletes presented substantially decreased amounts of physical activity (total counts per day), and time

in vigorous activities and MVPA during the COVID-19 pandemic, all of them accumulated the amount of MVPA suggested by the WHO [1] in both the pre-COVID (486.1 min/ day) and COVID-19 periods (392 min/day), which may be a protective factor in these athletes. According to the WHO [1], the increase in physical activity level, mainly moderate-vigorous activities, can improve the immune response 
Table 3 Comparison between quantity and quality of sleep during the pre-COVID and COVID-19 periods in young trained athletes

\begin{tabular}{lllll}
\hline Variables & pre-COVID & COVID & $p$ & $E S$ \\
\hline Sleep efficiency (\%) & $92.6(17)$ & $89.9(9.4)$ & 0.233 & 0.27 \\
Time in bed (hour/day) & $7.4 \pm 1.1$ & $8.0 \pm 1.2$ & 0.103 & 0.54 \\
Sleep time (hour/day) & $6.7 \pm 1.2$ & $6.9 \pm 1.2$ & 0.454 & 0.17 \\
WASO (total/day) & $19.6(69)$ & $41.8(47.8)$ & 0.078 & 0.61 \\
Latency (min/day) & $0.1(5.4)$ & $0.7(9)$ & 0.859 & 0.17 \\
\hline
\end{tabular}

WASO wake after sleep onset (total per day), ES effect size. Values are mean \pm standard deviation or median (interquartile range)

to infections, which would be very important during the pandemic period [34]. Martínez-de-Quel et al. [35] agreed in part with our data, since the authors analyzed the impact of mandatory confinement in students over 18 years old using the Minnesota Leisure Time Physical Activity Questionnaire to measure PA and the Pittsburgh Sleep Quality Index to verify the sleep quality. The authors demonstrated that a total of 48 days locked down at home had a negative impact on PA and sleep quality. In the present study, after about 90 days locked down at home, the athletes also presented decreased PA levels and increased ST. However, there is no updated scientific information about the amount of physical activity that may prevent a decrease in athlete performance and future studies should investigate the chronic effect of ST on performance.

Although no significant differences were found in quantity and quality of sleep, when analyzing the hand-written sleep diary that each athlete recorded, the athletes usually went to bed $\sim 2 \mathrm{~h}$ later and woke up $\sim 3 \mathrm{~h}$ later during the COVID period compared to the pre-COVID period. With regard to the quantity and quality of sleep, Kent et al. [15] showed that a social isolation routine could lead to sleep disorders, anxiety, and depression. Sleep quality has huge importance for athletes, since it enables maintained alertness, contributes to a shorter reaction time, and improves mood and physical vigor [20,36]. Furthermore, Rowlands et al. [37] used data from 91,248 UK Biobank participants with accelerometer data and demonstrated an association between markers of sleep/rest and physical activity and the risk or severity of COVID-19 infection. Therefore, the prevention of changes in sleep and PA behavior seem to be very important for the whole population and it is necessary to investigate whether a long period of quarantine could impact sleep quality in athletes.

Despite the importance of this study, some limitations should be mentioned such as, performance analyses were not carried out, a small number of athletes were evaluated, and there were no subjective measurements of sleep and PA, which could contribute to greater understanding of the routine of athletes. However, it is necessary to highlight that these athletes were evaluated objectively at baseline (preCOVID period), which was 2 weeks before the main competition for these athletes in 2019 and 1 year later during lockdown (June 2020), using an accelerometer instead of questionnaires or an online survey for the second evaluation, which present some limitations based on recall bias, such as poor self-perception of PA intensity. However, no gold standard measurements were used, such as polysomnography for sleep or calorimetric chamber for PA.

Regarding practical applications, coaches and sports physiologists could use these results to improve their exercise prescription, according to levels of habitual PA, ST, and sleep quantity and quality of badminton athletes during the COVID-19 pandemic period.

\section{Conclusion}

In conclusion, young badminton athletes presented increased ST, and decreased total PA, time in MVPA, and time in vigorous PA during the COVID-19 pandemic compared to the pre-COVID period, however, there were no significant differences for sleep parameters. Therefore, future longitudinal studies should be conducted to investigate the chronic effects of quarantine on morphological, physiological, and psychological variables in athletes. 

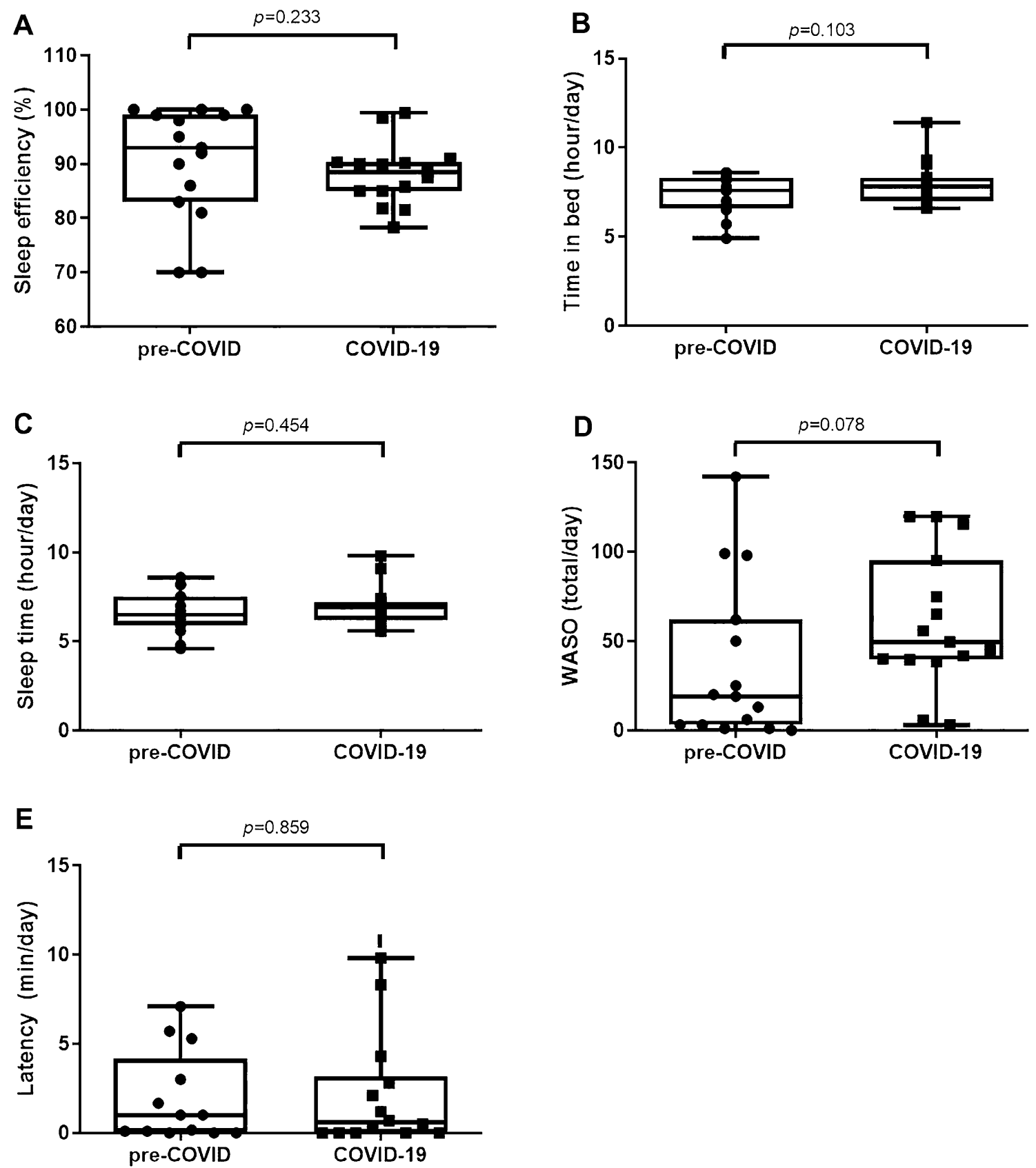

Fig. 2 Comparison between quantity and quality of sleep during the pre-COVID and COVID-19 periods in young trained athletes. a Sleep efficiency (\%) in median and interquartile range; b Time in bed (hour/ day) in mean and standard deviation; c Sleep time (hour/day) in mean

and standard deviation; $\mathbf{d}$ WASO = wake after sleep onset (total/day) in median and interquartile range; e Latency (min/day) in median and interquartile range

Acknowledgements We thank Dr. Kong Chen for the use of the Funding The authors would like to thank FAPEPI for their support accelerometers (funded by NIH Z01 DK071013), and all athletes and coaches from Brazilian Badminton Confederation and Associação de (FAPEPI/MCT/CNPq No. 007/2018). 


\section{Declarations}

Conflicts of interests The authors declare that they have no conflict of interest.

Ethics approval All procedures performed were developed according to the Declaration of Helsinki and approved by the Research Ethics Committee at the Federal University of Piauí (protocol: 2.552.506).

Informed consent Informed consent was obtained from all individual participants included in the study.

\section{References}

1. Bull FC, Al-Ansari SS, Biddle S et al (2020) World Health Organization 2020 guidelines on physical activity and sedentary behaviour. Br J Sports Med 54:1451-1462

2. Izzicupo P, Di Baldassarre A, Ghinassi B, Reichert FF, Kokubun E, Nakamura FY (2019) Can off-training physical behaviors influence recovery in athletes? A scoping review. Front Physiol 10:1

3. Wu XY, Han LH, Zhang JH, Luo S, Hu JW, Sun K (2017) The influence of physical activity, sedentary behavior on healthrelated quality of life among the general population of children and adolescents: a systematic review. PLoS ONE 12:e0187668

4. World Health Organization (2019) Global action plan on physical activity 2018-2030: more active people for a healthier world. World Health Organization, Geneva

5. Sperlich B, Becker M, Hotho A et al (2017) Sedentary behavior among national elite rowers during off-training — a pilot study. Front Physiol 8:655

6. Tremblay MS, LeBlanc AG, Kho ME et al (2011) Systematic review of sedentary behaviour and health indicators in schoolaged children and youth. Int J Behav Nutr Phys Act 8:98

7. Weiler R, Aggio D, Hamer M, Taylor T, Kumar B (2015) Sedentary behaviour among elite professional footballers: health and performance implications. Bmj Open Sport Exerc Med 1:1-4

8. Constandt B, Thibaut E, De Bosscher V, Scheerder J, Ricour M, Willem A (2020) Exercising in times of lockdown: an analysis of the impact of COVID-19 on levels and patterns of exercise among adults in Belgium. Int J Environ Res Public Health 17:4144

9. Chirico A, Lucidi F, Galli F, Giancamilli F, Vitale J, Borghi S et al (2020) COVID-19 outbreak and physical activity in the Italian population: a cross-sectional analysis of the underlying psychosocial mechanisms. Front Psychol 11:2100

10. Carmody S, Murray A, Borodina M, Gouttebarge V, Massey A (2020) When can professional sport recommence safely during the COVID-19 pandemic? Risk assessment and factors to consider. Br J Sports Med 54:946-948

11. López-Valenciano A, Suárez-Iglesias D, Sanchez-Lastra MA, Ayán C (2021) Impact of COVID-19 pandemic on university students' physical activity levels: an early systematic review. Front Psychol 15:624567

12. Stockwell S, Trott M, Tully M, Shin J, Barnett Y, Butler L et al (2021) Changes in physical activity and sedentary behaviours from before to during the COVID-19 pandemic lockdown: a systematic review. BMJ Open Sport Exerc Med 7:e000960

13. Mujika I, Padilla S (2000) Detraining: Loss of training-induced physiological and performance adaptations. Part II: Long term insufficient training stimulus. Sports Med 30:145-154

14. Paoli A, Musumeci G (2020) Elite athletes and COVID-19 lockdown: future health concerns for an entire sector. J Funct Morphol Kinesiol 30:1
15. Kent de Grey RG, Uchino BN, Trettevik R, Cronan S, Hogan JN (2018) Social support and sleep: a meta-analysis. Health Psychol 37:787-798

16. Kelley GA, Kelley KS (2017) Exercise and sleep: a systematic review of previous meta-analyses. J Evid Based Med 10:26-36

17. Hartescu I, Morgan K, Stevinson CD (2015) Increased physical activity improves sleep and mood outcomes in inactive people with insomnia: a randomized controlled trial. J Sleep Res 24:526-534

18. Mead MP, Baron K, Sorby M, Irish LA (2019) Daily associations between sleep and physical activity. Int J Behav Med 26:562-568

19. Yang Y, Shin JC, Li D, An R (2017) Sedentary behavior and sleep problems: a systematic review and meta-analysis. Int J Behav Med 24:481-492

20. Mah CD, Mah KE, Kezirian EJ, Dement WC (2011) The effects of sleep extension on the athletic performance of collegiate basketball players. Sleep 34:943-950

21. Lastella M et al (2014) Sleep/wake behaviours of elite athletes from individual and team sports. Eur J Sport Sci 15:94-100

22. Vitale JA, La Torre A, Banfi G (2019) If RAR's acrophase is influenced by the sport discipline, how actigraphy-based sleep parameters vary in triathlon, volleyball and soccer athletes? Chronobiol int 36:735-738

23. Bonato M, La Torre A, Saresella M, Marventano I, Merati G, Banfi G et al (2020) Effect of high-intensity interval training versus small-sided games training on sleep and salivary cortisol level. Int J Sports Physiol Perform 15:1237-1244

24. Nédélec M, Halson S, Abaidia AE, Ahmaidi S, Dupont G (2015) Stress, sleep and recovery in elite soccer: a critical review of the literature. Sports Med 45:1387-1400

25. Freedson PS, Melanson E, Sirard J (1998) Calibration of the computer science and Applications Inc. accelerometer. Med Sci Sports Exerc 30:777-781. https://doi.org/10.1097/00005768-19980 5000-00021

26. Crouter SE, Flynn JI, Bassett DR Jr (2015) Estimating physical activity in youth using a wrist accelerometer. Med Sci Sports Exerc 47:944

27. Slater JA, Botsis T, Walsh J, King S, Straker LM, Eastwood PR (2015) Assessing sleep using hip and wrist actigraphy. Sleep Biol Rhythms 1:172-180

28. Leeder J, Glaister M, Pizzoferro K, Dawson J, Pedlar C (2012) Sleep duration and quality in elite athletes measured using wristwatch actigraphy. J Sports Sci 30:541-545

29. Staunton C, Gordon B, Custovic E, Stanger J, Kingsley M (2017) Sleep patterns and match performance in elite Australian basketball athletes. J Sci Med Sport 20:786-789

30. Phomsoupha M, Laffaye G (2015) The science of badminton: game characteristics, anthropometry, physiology, visual fitness and biomechanics. Sports Med 45:473-495

31. Tomaszewski P, Kęska A, Tkaczyk J, Nowicki D, SienkiewiczDianzenza E (2017) Somatic characteristics and motor fitness of elite and sub-elite Polish male badminton players. J Sports Med Phys Fitness 58:1456-1464

32. Pillay L, Van Rensburg DCCJ, Van Rensburg AJ et al (2020) Nowhere to hide: The significant impact of coronavirus disease 2019 (COVID-19) measures on elite and semi-elite South African athletes. J Sci Med Sport 23:670-679

33. Thorp AA, Kingwell BA, Sethi P, Hammond L, Owen N, Dunstan DW (2014) Alternating bouts of sitting and standing attenuate postprandial glucose responses. Med Sci Sports Exerc 46:2053-2061

34. Simpson RJ, Katsanis E (2020) The immunological case for staying active during the COVID-19 pandemic. Brain Behav Immun 4:1

35. Martínez-de-Quel Ó, Suárez-Iglesias D, López-Flores M, Pérez CA (2021) Physical activity, dietary habits and sleep quality 
before and during COVID-19 lockdown: a longitudinal study. Appetite 158:105019

36. Bleyer FTS, Barbosa DG, Andrade RD, Teixeira CS, Felden ÉPG (2015) Sleep and musculoskeletal complaints among elite athletes of Santa Catarina. Rev Dor 16:102-108

37. Rowlands AV, Kloecker DE, Chudasama Y et al (2021) Association of timing and balance of physical activity and rest/sleep with risk of COVID-19: a UK Biobank Study. Mayo Clin Proc 96:156-164

Publisher's Note Springer Nature remains neutral with regard to jurisdictional claims in published maps and institutional affiliations. 